Revue de droit comparé du travail et de la sécurité sociale

3 | 2021

Droits du travail et systèmes nationaux de protection sociale au prisme de la crise sanitaire. Adaptations ou changements profonds?

\title{
République de Serbie
}

Filip Bojić

\section{(2) OpenEdition}

\section{Journals}

Édition électronique

URL : https://journals.openedition.org/rdctss/2218

DOI : $10.4000 /$ rdctss.2218

ISSN : 2262-9815

Traduction(s) :

Changes and amendments to the social legislation of the Republic of Serbia - URL : https:// journals.openedition.org/rdctss/2773 [en]

\section{Éditeur}

Centre de droit comparé du travail et de la sécurité sociale

\section{Édition imprimée}

Date de publication : 1 novembre 2021

Pagination : 208-211

ISSN : $2117-4350$

\section{Référence électronique}

Filip Bojić, "République de Serbie », Revue de droit comparé du travail et de la sécurité sociale [En ligne]

3 | 2021, mis en ligne le 01 novembre 2021, consulté le 15 novembre 2022. URL : http:// journals.openedition.org/rdctss/2218; DOI : https://doi.org/10.4000/rdctss.2218

\section{(c) (i) (3)}

Creative Commons - Attribution - Pas d'Utilisation Commerciale - Pas de Modification 4.0 International - CC BY-NC-ND 4.0

https://creativecommons.org/licenses/by-nc-nd/4.0/ 


\title{
FILIP BOJIĆ
}

\author{
UNIVERSITÉ DE BELGRADE
}

Au premier semestre de 2021, l'Assemblée nationale de la République de Serbie a adopté plusieurs amendements dans le domaine de la sécurité sociale qui concernent plus particulièrement la Loi sur l'assurance retraite et invalidité (I) et la Loi sur l'aide financière pour des familles avec des enfants (II). Il convient de signaler que la Loi sur l'emploi et l'assurance-chômage a également subi de nombreux amendements, qui ne régissent pas l'assurance-chômage mais règlent le domaine de l'emploi, raison pour laquelle ils ne seront pas analysés dans le présent article.

\section{I - AMENDEMENTS À LA LOI SUR L'ASSURANCE RETRAITE ET INVALIDITÉ}

Par rapport aux modifications précédentes de la Loi sur l'assurance retraite et invalidité qui ont finalement sécurisé, sous certaines conditions, le droit à une pension de survie pour les conjoints non mariés, ces nouveaux amendements ont été adoptés pour préciser certaines normes juridiques déterminant le montant de la pension de vieillesse anticipée.

La pension de vieillesse anticipée a été réaffirmée dans la législation serbe en 2014. La Loi sur l'assurance retraite et invalidité régit précisément les conditions dans lesquelles un assuré peut exercer le droit à la pension de vieillesse anticipée, et comprend les règles déterminant le montant de la pension de vieillesse anticipée ${ }^{1}$. Sur ce point, le législateur a précisé que ce montant était déterminé de la même manière que celui de la pension de vieillesse, à l'exception du fait que le montant de la pension de vieillesse anticipée est réduit de façon graduelle et permanente à hauteur de $-0,34 \%$ pour chaque mois avant 65 ans $^{2}$. En revanche, ce montant ne peut pas être réduit plus de 20,4\%. L'assuré qui décide d'exercer une demande de droit à la pension de vieillesse anticipée percevra le montant ainsi déterminé jusqu'à la fin de sa vie. Cette règle n'a pas emporté l'approbation du public et, à plusieurs reprises, les représentants de différentes associations de retraités et de syndicats ont demandé les modifications de cette disposition.

Il faut par ailleurs garder à l'esprit qu'au cours des premières années suivant la mise en $œ u v r e$ de cette disposition, peu d'assurés ont finalement décidé d'exercer leur droit à la pension de vieillesse anticipée ${ }^{3}$. Ceci tient aux conditions très strictes d'octroi, notamment celles relatives à la durée d'assurance (40 ans de service), mais aussi à la réduction graduelle du montant de la pension de vieillesse anticipée.

1 Pour plus de détails sur ce sujet, voir Revue de droit comparé du travail et de la sécurité sociale, 2014/1, p. 170.

2 Loi sur l'assurance retraite et invalidité, (Journal officiel de la République de Serbie, $n^{\circ} 34 / 2003$; Modifications législatives $n^{\circ} 64 / 2004, n^{\circ} 84 / 2004, n^{\circ} 85 / 2005, n^{\circ} 101 / 2005, n^{\circ} 63 / 2006, n^{\circ} 5 / 2009$, $n^{\circ} 107 / 2009, n^{\circ} 101 / 2010, n^{\circ} 93 / 2012, n^{\circ} 62 / 2013, n^{\circ} 108 / 2013, n^{\circ} 75 / 2014, n^{\circ} 142 / 2014, n^{\circ} 73 / 2018$, $n^{\circ} 46 / 2019, n^{\circ} 86 / 2019, n^{\circ} 62 / 2021$, art.70a

3 Selon les données statistiques du Fonds de la République pour l'assurance pension et invalidité, 17900 assurés ont décidé d'exercer leur droit à la pension de vieillesse anticipée entre 2014 et 2018. 


\section{République de Serbie}

La nouveauté législative adoptée en 2021 n'a pas répondu aux demandes exprimées par les syndicats et associations de retraités mécontents, dans la mesure où elle ne prévoit qu'une seule exception relative à la détermination du montant de la pension de vieillesse anticipée au profit des assurés qui étaient déjà bénéficiaires du droit à l'indemnité particulière $e^{4}$. Pour ces assurés, le montant de la pension de vieillesse anticipée est calculé de la même manière que le montant de la pension de vieillesse, et donc cette catégorie ne se verra pas réduire le montant conformément aux règles applicables aux assurés qui décident de déposer une demande de droit à la pension de vieillesse anticipée ${ }^{5}$.

L'autre modification importante concerne le montant de la pension de survie pour une catégorie particulière de titulaires, les veufs et veuves d'un militaire professionnel décédé lors du service. Ils exerceront leur droit à la pension de survie, quel que soit lâage légal prescrit, mais à condition de ne pas se remarier ${ }^{6}$. II convient de noter que, dans la législation serbe, le remariage d'un bénéficiaire de la pension de survie ne constitue pas un motif de résiliation du droit à pension, sauf dans le cas susmentionné.

Les nouvelles modifications législatives ont également introduit une autre exception pour cette catégorie des bénéficiaires, concernant la détermination du montant de la pension de survie. En général, si un membre de la famille exerce son droit à la pension de survie, le montant de cette prestation est équivalent à $70 \%$ du montant de la pension dont aurait bénéficié l'assuré au moment du décès. En revanche, le montant de la pension de survie pour le conjoint d'un militaire professionnel décédé lors du service est équivalent à $100 \%$ de la pension à laquelle aurait eu droit l'assuré décédé au moment du décès ${ }^{7}$. Ces nouveautés renforcent le concept établi de longue date de compensation sociale pour les familles des militaires qui ont perdu la vie au cours de leur service.

\section{II - LES AMENDEMENTS À LA LOI SUR L'AIDE FINANCIÈRE POUR DES FAMILLES AVEC DES ENFANTS}

Les amendements adoptés de la Loi sur l'aide financière pour des familles avec des enfants sont la conséquence du fort mécontentement du public à l'égard des nouvelles dispositions législatives sur la protection des familles et des enfants. La Loi sur l'aide financière aux familles avec enfants a été adoptée en 2017 et a commencé à être appliquée le 1 erjuillet 2018. L'objectif principal de ce texte était d'apporter une aide financière aux familles avec enfants et visait à améliorer les conditions de satisfaction des besoins essentiels des enfants, harmoniser le travail et la parentalité, encourager les taux de natalité ou soutenir les parents pour atteindre le nombre d'enfants souhaité, améliorer la situation financière des familles avec des enfants handicapés ou nécessitant des soins. Néanmoins, force est de constater que le texte de loi contient un certain nombre de solutions défavorables qui remettent en cause son objectif fondamental.

De plus, la Cour constitutionnelle de la République de Serbie a, dans quelques décisions, déclaré inconstitutionnelles certaines dispositions de cette Loi. C'est pourquoi, il

4 Loi sur l'assurance retraite et invalidité, art. 70d.

5 Explication des amendements de la Loi sur l'assurance retraite et invalidité de 2021, p. 4 : $\underline{\text { http:// }}$ www.parlament.gov.rs/upload/archive/files/cir/pdf/predlozi zakona/2021/934-21.pdf

6 Loi sur l'assurance retraite et invalidité, art. 30a.

7 Loi sur l'assurance retraite et invalidité, art. 71. 
était nécessaire d'harmoniser ces dispositions avec la Constitution, c'est pourquoi plusieurs amendements ont été adoptés. À l'instar des amendements analysés ci-avant à la loi sur l'assurance pension et invalidité, un certain nombre de modifications apportées à la loi sur l'aide financière aux familles avec enfants sont liées à la détermination du montant de l'indemnisation pour les revenus versés pendant le congé de maternité, le congé pour s'occuper d'un enfant et les absences du travail pour la garde spéciale d'enfants. En ce qui concerne le montant maximum de la compensation mensuelle du salaire de base, il est prévu qu'à partir du $1^{\text {er }}$ janvier 2022, le montant à payer au bénéficiaire ne puisse dépasser cinq salaires mensuels moyen en République de Serbie, calculés selon les dernières données publiées de l'Autorité nationale chargée de la statistique le jour du début de l'exercice des droits 8 .

Il faut noter que cette solution figurait dans la Loi précédente sur l'aide financière aux familles avec enfants et que l'adoption de la nouvelle loi prévoit que le montant supérieur à trois salaires mensuels moyens en République de Serbie ne peut pas être versé à un bénéficiaire du droit à l'indemnité. Ainsi, certains bénéficiaires parmi les hauts revenus avant d'exercer leur droit au congé de maternité ou au congé du travail pour garde d'enfant, se sont trouvés dans une position défavorable. Il semble que les nouveaux amendements adoptés aient corrigé ce défaut.

La deuxième modification est semblable à la première sauf qu'elle se réfère au montant du salaire minimum versé pendant le congé. Il est en effet prévu que le montant total mensuel de l'indemnité pendant le congé de maternité ne puisse pas être inférieur au salaire minimum déterminé le jour du début de l'exercice de ce droit. Le montant du salaire minimum est calculé en fonction du salaire horaire minimum, déterminé conformément à la loi, puis multiplié par 184 heures et augmenté des impôts et cotisations correspondants.

Cette solution représente une amélioration significative en comparaison avec la solution précédente, qui désavantageait certains bénéficiaires étant donné que la loi prévoyait que " le montant total mensuel de l'indemnité salariale pendant le congé de maternité ne peut être inférieur au SMIC tel que fixé le jour du dépôt de la demande, si l'autorité compétente a relevé au moins six salaires minimum sur lesquels les cotisations sur le revenu ont été versées $»^{9}$.

La Cour constitutionnelle de Serbie luz-247/2018 a considéré que cette disposition de la Loi sur l'aide financière aux familles avec enfants était non conforme à la Constitution et aux Traités internationaux ratifiés par la Serbie.

La Cour constitutionnelle a en effet déclaré que ladite disposition était en contradiction avec l'article de la Constitution, qui garantit un soutien spécial aux mères avant et après l'accouchement, mais aussi avec la Convention n¹83 sur la protection de la maternité de l'Organisation internationale du travail, qui prévoit que le montant de l'indemnisation doit être tel qu'il assure le maintien de la bonne santé des femmes et des enfants selon un

8 Loi sur l'aide financière pour des familles avec des enfants (Journal officiel de la République de Serbie, n¹13/2017 ; Modifications législatives $n^{\circ} 50 / 2018, n^{\circ} 46 / 2021, n^{\circ} 51 / 2021, n^{\circ} 53 / 2021$, $\left.n^{\circ} 66 / 2021\right)$, art. 13, acte 4.

9 Loi sur l'aide financière pour des familles avec des enfants, art. 14. 


\section{Républioue de SERBie}

niveau de vie convenable ${ }^{10}$. Etant donné que, dans certains cas, il avait été enregistré moins de six salaires minimum, le montant de la prestation était inférieur au salaire minimum et ne pourrait pas, comme indiqué dans la Convention, assurer un niveau de vie convenable. De ce fait, les modifications de la Loi furent nécessaires pour corriger cette lacune dans les meilleurs délais.

En outre, un amendement a également été apporté afin d'harmoniser cette loi avec les autres dispositions relatives au domaine de la sécurité sociale et à la question de versement des prestations sociales. Cet amendement prévoit que le bénéficiaire auquel la prestation est versée alors qu'il n'en avait pas le droit est tenu de restituer les sommes perçues au ministère chargé de l'aide financière aux familles avec enfants ${ }^{11}$. Le même article prévoit que le ministère aura droit au remboursement des impôts et cotisations versés au titre de l'assurance sociale obligatoire.

Enfin, il faut souligner que, depuis octobre 2020, la Loi sur les ministères a créé un ministère spécial chargé de la protection de la famille et de la démographie. Ainsi, la question relative à l'aide financière aux familles avec des enfants ne relève plus de la compétence juridictionnelle du ministère chargé de affaires sociales, ce qui est aussi corrigé dans tous les articles de la loi où la compétence du ministère était mentionnée.

10 La décision de la Cour constitutionnelle luz-247/2018. II convient de souligner que la décision de la Cour constitutionnelle a trouvé une disharmonie entre les dispositions de la Loi sur l'aide financière pour des familles avec des enfants, ensuite avec les dispositions de la Loi sur le travail et de la Loi sur l'assurance maladie, qui disposent que le montant de l'indemnité en cas d'incapacité temporaire de travail ne peut pas être inférieur au montant du salaire minimum

11 Loi sur l'aide financière pour des familles avec des enfants, art. 44a. 